\title{
INDUCED FIBRATIONS AND COFIBRATIONS $\left({ }^{1}\right)$
}

\author{
BY \\ T. GANEA
}

Introduction. It is well known that any map is homotopically equivalent to a fiber map, i.e., to the projection of the total space on the base in a fibration. Simple examples, however, reveal that there are maps which fail to be homotopically equivalent to any inclusion of a fiber in the total space, and the problem of characterizing the maps which are equivalent to such inclusions was first raised in [14]. By the first remark, the map under consideration may be assumed from the beginning to be a fiber map $p: E \rightarrow B$, and the problem is thus converted into that of characterizing the fibrations $p$ which are equivalent to induced fibrations. There are two immediate necessary conditions: the fiber $F$ of $p$ must have the homotopy type of some loop space $\Omega Y$, and the inclusion $i: F \rightarrow E$ must map the generalized homotopy group $\pi(X, \Omega F)$ into the center of $\pi(X, \Omega E)$ for any space $X$; the latter is a mild generalization of the well known fact that the boundary operator in the homotopy sequence of a fibration $E \rightarrow B \rightarrow Y$ maps $\pi_{2}(Y)$ into the center of $\pi_{1}(E)$. The first result in this direction is due to Serre and asserts that $p$ is an induced fibration if $B$ is 1 -connected and $F$ has a single nonvanishing (Abelian) homotopy group. This result was generalized [6], [12], [17], [15] to allow $F$ to have at most $m-1$ nonvanishing homotopy groups in consecutive dimensions provided it has the homotopy type of a loop space and $B$ is $(m-1)$-connected. In the first two sections of this paper we give results which allow $F$ to have $2 m-1$ nonvanishing homotopy groups in consecutive dimensions provided $B$ is $(m-1)$-connected and $F$ has the homotopy type of a loop space under a homotopy equivalence fulfilling a certain condition which involves the "operation" of $\Omega B$ on $F$. In case $F$ has $m$ nonvanishing homotopy groups in consecutive dimensions, the sufficient condition in order that $p$ be induced is expressed by means of the vanishing of a certain Whitehead product; this result answers a question raised in [15]. Dually, any map is homotopically equivalent to an inclusion $A \rightarrow X$ having the homotopy extension property, but few maps are equivalent to identification maps resulting by shrinking to a point such a subset $A$ of $X$. This leads to the problem of characterizing the cofibrations $A \rightarrow X$ which are induced by maps of some space $Y$ into $A$. Our results extend the range of applicability of some previous results [8], [12] by imposing an additional condition which involves the "cooperation" of the suspension $\Sigma A$ on the cofiber $C$ obtained from $X$ by shrinking $A$ to a point; the condition is conveniently expressed in terms of the Hopf invariant of a cofibration

Presented to the Society, April 4, 1966; received by the editors April 7, 1966.

(1) This work was partially supported by N.S.F. GP-3902. 
discussed in [7, §4]. As an application, we prove by means of induced cofibration techniques the main result in [4] which asserts that an $(n-1)$-connected $H^{\prime}$-space $X$ of dimension $\leqq 3 n-3$ is equivalent to a suspension; our proof eliminates the assumption made in [4] that the homology of $X$ is finitely generated.

In principle, the methods used in this paper could be developed so as to yield results even if the fiber $F$ had more than $2 m-1$ nonvanishing homotopy groups or if, dually, the cofiber $C$ had larger dimension than allowed in the theorems below. However, the degree of complexity of the additional sufficient conditions which then appear is rapidly increasing and the results obtained do not seem to lead to effective computations.

The author is grateful to I. Namioka for helpful discussions, and to the referee for several suggestions which have led to improvements in the presentation.

1. Induced fibrations. All spaces in this paper are provided with a base-point denoted by $*$, and all maps and homotopies are assumed to preserve base-points. A triple

$$
\mathscr{F}: F \stackrel{i}{\longrightarrow} E \stackrel{p}{\longrightarrow} B
$$

is a fibration if $p$ has the covering homotopy property for all spaces and $F=p^{-1}(*)$; $i$ is the inclusion map. For any map $f: X \rightarrow Y$ we denote by $Y \cup_{f} C X$ the space obtained by attaching to $Y$ via $f$ the reduced cone over $X$; however, the subscript $f$ will frequently be omitted. A point in $C X$ is denoted by $s x(s \in I, x \in X)$; the map $x \rightarrow 1 x$ embeds $X$ as base in the cone, and $0 X \cup I *$ is identified to the vertex. Consider the diagram

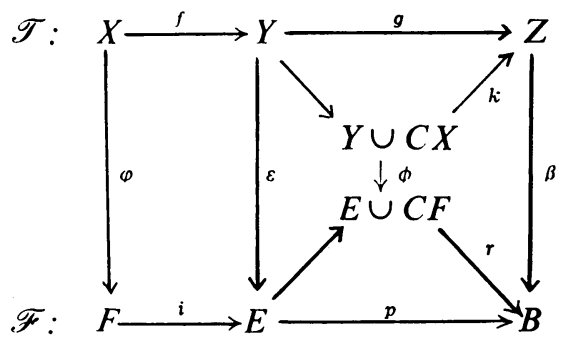

where $\mathscr{T}$ is any triple with $g \circ f=*, \mathscr{F}$ is a fibration, and $k$ and $r$ extend $g$ and $p$ by mapping the cones to the base-points. We shall repeatedly use the

Lemma 1.1. Suppose $h_{t}: X \rightarrow E$ is a homotopy satisfying $h_{0}=i \circ \varphi, h_{1}=\varepsilon \circ f$, and let $\phi$ be induced by $\varepsilon, \phi, h_{t}$. If there is a map $\beta$ such that $r \circ \phi \simeq \beta \circ k$, then there are maps $\varphi_{1}$ and $\varepsilon_{1}$ satisfying $\varphi_{1} \simeq \varphi, \varepsilon_{1} \simeq \varepsilon$, and yielding strict commutativity in the diagram

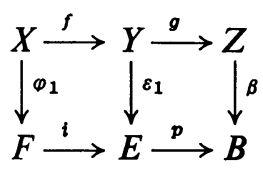


Proof. Let $M$ be the reduced mapping cylinder of $f$, let $j: X \rightarrow M$ be the standard embedding given by $j(x)=(0, x)$, and let $q: M \rightarrow Y \cup C X$ shrink to a point the subset $j(X)$ of $M$. The map $\phi$ is given [19] by

$$
\phi(y)=\varepsilon(y) \text { and } \begin{aligned}
\phi(s x) & =2 s \varphi(x) \quad \text { if } \quad 0 \leqq 2 s \leqq 1 \\
& =h_{2 s-1}(x) \text { if } 1 \leqq 2 s \leqq 2 .
\end{aligned}
$$

Then, $p \circ \mu=r \circ \phi \circ q$ provided $\mu: M \rightarrow E$ is given by

$$
\begin{aligned}
& \mu(1, y)=\varepsilon(y) \text { and } \mu(s, x)=i \circ \varphi(x) \quad \text { if } \quad 0 \leqq 2 s \leqq 1 \text {, } \\
& =h_{2 s-1}(x) \text { if } 1 \leqq 2 s \leqq 2 .
\end{aligned}
$$

Let $H_{t}: Y \cup C X \rightarrow B$ satisfy $H_{0}=r \circ \phi, H_{1}=\beta \circ k$. Since $p$ has the covering homotopy property, there is a homotopy $\mu_{t}: M \rightarrow E$ with $\mu_{0}=\prime \prime, p \circ \mu_{t}=H_{t} \circ q$. Define $\varepsilon_{1}=\mu_{1} \mid 1 \times Y$. Then, $\varepsilon_{1} \simeq \varepsilon$ and $p \circ \varepsilon_{1}=\beta \circ g$. Define

$$
\begin{aligned}
d_{t}: X \rightarrow E \text { by } \quad d_{t}(x) & =\mu_{2 t}(0, x) & \text { if } \quad 0 \leqq 2 t \leqq 1, \\
& =\mu_{1}(2 t-1, x) & \text { if } \quad 1 \leqq 2 t \leqq 2
\end{aligned}
$$

Since $q \circ j=*$ and $k(C X)=*$, one has $p \circ d_{t}=*$ and there results a homotopy $\varphi_{t}: X \rightarrow F$ with $i \circ \varphi_{t}=d_{t}$. Then, $i \circ \varphi_{1}=\varepsilon_{1} \circ f$ and $i \circ \varphi_{0}=i \circ \varphi$ so that $\varphi_{0}=\varphi$ and $\varphi_{1} \simeq \varphi$.

For any fibration $\mathscr{F}$ we may construct a sequence of fibrations

$$
\mathscr{F}_{k}: F_{k} \stackrel{i_{k}}{\longrightarrow} E_{k} \stackrel{p_{k}}{\longrightarrow} B \quad(k \geqq 0)
$$

as follows: $\mathscr{F}_{0}=\mathscr{F}, r_{k+1}: E_{k} \cup C F_{k} \rightarrow B$ extends $p_{k}$ by mapping the cone to the base-point, and $p_{k+1}$ results by converting $r_{k+1}$ into a homotopically equivalent fiber map. Let $X * Y$ be the join of $X$ and $Y$ taken as an identification space of the Cartesian product $X \times I \times Y$; a point in the join is denoted by $(1-s) x \oplus s y$ and $\frac{1}{2} * \oplus \frac{1}{2} *$ serves as base-point. The following fact is proved in [7, 1.1 and 1.2]:

Proposition 1.2. If $E$ and $B$ have the homotopy type of $C W$-complexes, then there is a homotopy equivalence $F_{k} * \Omega B \rightarrow F_{k+1}$.

CoRollary 1.3. Suppose that $B$ is $(m-1)$-connected, $F$ is $(n-1)$-connected, and that $\pi_{q}(Y) \neq 0$ only if $2 \leqq q \leqq n+(k+1) m-1(m \geqq 1, n \geqq 1)$. For any map $g: E_{k} \rightarrow Y$ with $g \circ i_{k} \simeq 0$ there is a map $f: B \rightarrow Y$ with $f \circ p_{k} \simeq g$.

Proof. Since $g \circ i_{k} \simeq 0, g$ extends to a map $h: E_{k} \cup C F_{k} \rightarrow Y$. The connectivity of the join and 1.2 imply that $r_{k+1}$ is $(n+(k+1) m)$-connected, and an obvious obstruction argument yields a map $f$ satisfying $f \circ r_{k+1} \simeq h$.

In any fibration $\mathscr{F}$ the loop space $\Omega B$ operates [5] on the fiber $F$ through a map $\rho: F \times \Omega B \rightarrow F$. The Hopf construction yields the composite

$$
H: F * \Omega B \stackrel{v}{\longrightarrow} \Sigma(F \times \Omega B) \stackrel{\Sigma \rho}{\longrightarrow} \Sigma F
$$


in which $\Sigma$ is the reduced suspension functor and $V$ shrinks to a point the two ends of the join and the segment through the base-point so that

$$
V((1-s) x \oplus s \omega)=\langle s,(x, \omega)\rangle .
$$

We describe two fibrations $\mathscr{F}$ and $\mathscr{F}^{\prime}$ over the same base as equivalent if there is a homotopy equivalence $\varepsilon: E \rightarrow E^{\prime}$ such that $p^{\prime} \circ \varepsilon=p$; there exists then a homotopy inverse $\varepsilon^{\prime}$ of $\varepsilon$ such that $p \circ \varepsilon^{\prime}=p^{\prime}$. A fibration $\mathscr{F}$ is induced if there is a space $Y$ and a map $f: B \rightarrow Y$ such that $\mathscr{F}$ is equivalent to the fibration strictly induced by $f$ from $\Omega Y \rightarrow P Y \rightarrow Y$, i.e., to the triple

$$
\Omega Y \stackrel{\imath}{\longrightarrow} W=\{(b, \eta) \in B \times P Y \mid f(b)=\eta(1)\} \stackrel{w}{\longrightarrow} B
$$

where $P Y$ is the space of paths in $Y$ emanating from *, $\Omega Y$ is the loop space, $l(\omega)=(*, \omega)$, and $w(b, \eta)=b$; it then follows that $F$ has the homotopy type of $\Omega Y$.

The map $R$ below is given by $R\langle s, \omega\rangle=\omega(s)$.

THEOREM 1.4. Let $\mathscr{F}: F \stackrel{i}{\longrightarrow} E \stackrel{p}{\longrightarrow} B$ be a fibration in which $E$ and $B$ have the homotopy type of $C W$-complexes. Suppose that $B$ is $(m-1)$-connected and that $\pi_{q}(F) \neq 0$ only if $n \leqq q \leqq n+2 m-2$, where $m \geqq 1$ and $n \geqq 1$. If there is a space $Y$ and $a$ homotopy equivalence $\theta: F \rightarrow \Omega Y$ such that the composite

$$
F * \Omega B \stackrel{H}{\longrightarrow} \Sigma F \stackrel{\Sigma \theta}{\longrightarrow} \Sigma \Omega Y \stackrel{R}{\longrightarrow} Y
$$

is nullhomotopic, then $\mathscr{F}$ is induced by some map $f: B \rightarrow Y$.

Proof. Consider the diagram

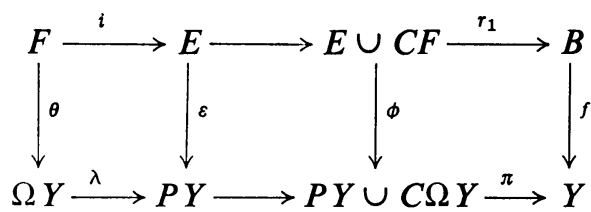

in which $\lambda$ is the inclusion, $\pi(\eta)=\eta(1)$ and $\pi(C \Omega Y)=*, \varepsilon(E)=*$, and $\phi$ is induced by $\varepsilon, \theta$, and any homotopy connecting $\lambda \circ \theta$ with $\varepsilon \circ i$. Let $\sigma: E \cup C F \rightarrow \Sigma F$ shrink $E$ to a point, and let $-\sigma$ stand for $\sigma$ followed by the map $\langle s, x\rangle \rightarrow\langle 1-s, x\rangle$; let $-\tau: P Y \cup C \Omega Y \rightarrow \Sigma \Omega Y$ be similarly defined. Then,

$$
\Sigma \theta \circ(-\sigma) \simeq(-\tau) \circ \phi \text { and } R \circ(-\tau) \simeq \pi .
$$

According to [7, 1.4], $H$ is homotopic to the composite

$$
F * \Omega B \stackrel{v}{\longrightarrow} F_{1} \stackrel{i_{1}}{\longrightarrow} E_{1} \stackrel{u}{\longrightarrow} E \cup C F \stackrel{-\sigma}{\longrightarrow} \Sigma F
$$

in which $v$ is the homotopy equivalence in 1.2 and $u$ is the homotopy equivalence obtained when converting $r_{1}$ into $p_{1}$ so that $p_{1} \simeq r_{1} \circ u$. Therefore, it follows from (3) and (2) that $\pi \circ \phi \circ u \circ i_{1} \circ v \simeq 0$, hence $\pi \circ \phi \circ u \circ i_{1} \simeq 0$, and 1.3 with $k=1$ 
yields a map $f$ satisfying $f \circ p_{1} \simeq \pi \circ \phi \circ u$, hence $f \circ r_{1} \simeq \pi \circ \phi$. As a consequence, 1.1 yields the strictly commutative diagram on the left
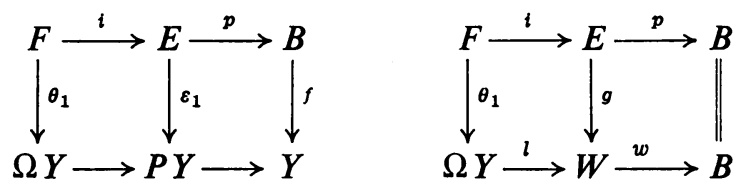

with $\theta_{1} \simeq \theta$, and the result follows upon applying the five lemma for homotopy groups in the strictly commutative diagram on the right, where $g(a)=\left(p(a), \varepsilon_{1}(a)\right)$.

We derive the often proved [6], [12], [17], [15]

COROLlaRY 1.5. If $B$ is $(m-1)$-connected and $\pi_{q}(F) \neq 0$ only if $n \leqq q \leqq n+m-2$, where $m \geqq 2$ and $n \geqq 1$, and if there is a homotopy equivalence $\theta: F \rightarrow \Omega Y$, then $\mathscr{F}$ is induced by some map $f: B \rightarrow Y$.

Proof. $F * \Omega B$ is $(n+m-1)$-connected and $\pi_{q}(Y)=0$ if $q \geqq n+m$ so that (2) is certainly nullhomotopic.

REMARK 1.6. The homotopy equivalence $F \rightarrow \Omega Y$ resulting in 1.4 and 1.5 from the fact that $\mathscr{F}$ is induced by $f: B \rightarrow Y$ is homotopic to the original homotopy equivalence $\theta: F \rightarrow \Omega Y$.

Note that 1.5 is trivially true if $m=1: F$ is then contractible, $p$ is a homotopy equivalence, and $\mathscr{F}$ is induced by the map $B \rightarrow *$.

In the preceding arguments we have implicitly used the well-known fact (see e.g. [17]) that $F$ has the homotopy type of a CW-complex if $E$ and $B$ do. Actually, the following slightly stronger result holds:

Proposition 1.7. Let $\mathscr{F}$ be a fibration in which $E$ and $B$ have the homotopy type of $C W$-complexes. Then, there exists a strictly commutative diagram

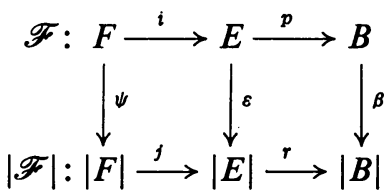

in which $\psi, \varepsilon, \beta$ are homotopy equivalences, the bottom row is a fibration, $|B|$ is a $C W$-complex, and $(|E|,|F|)$ has the homotopy type of a $C W$-pair.

Proof. Select homotopy equivalences $\varepsilon^{\prime}: E \rightarrow E^{\prime}$ and $\beta^{\prime}: B \rightarrow B^{\prime}$, where $E^{\prime}$ and $B^{\prime}$ are $C W$-complexes. There exists a cellular map $p^{\prime}: E^{\prime} \rightarrow B^{\prime}$ with $p^{\prime} \circ \varepsilon^{\prime} \simeq \beta^{\prime} \circ p$. Its reduced mapping cylinder $|B|$ is a CW-complex in which $E^{\prime}$ and $B^{\prime}$ are embedded as subcomplexes by inclusions $p^{\prime \prime}: E^{\prime} \rightarrow|B|$ and $\beta^{\prime \prime}: B^{\prime} \rightarrow|B|$ satisfying $p^{\prime \prime} \simeq \beta^{\prime \prime} \circ p^{\prime}$. Since $\beta^{\prime \prime}$ is a homotopy equivalence, so is the composite $\beta=\beta^{\prime \prime} \circ \beta^{\prime}$. Let

$$
|E|=\left\{\lambda \in|B|^{I} \mid \lambda(1) \in p^{\prime \prime}\left(E^{\prime}\right)\right\}
$$

be the mapping track of $p^{\prime \prime}$, and define $\varepsilon^{\prime \prime}\left(a^{\prime}\right)(s)=p^{\prime \prime}\left(a^{\prime}\right), r(\lambda)=\lambda(0)$. Then, $\varepsilon^{\prime \prime}: E^{\prime} \rightarrow|E|$ is a homotopy equivalence, $r$ is a fiber map, and $r \circ \varepsilon^{\prime \prime}=p^{\prime \prime}$. One has 
$r \circ \varepsilon^{\prime \prime} \circ \varepsilon^{\prime} \simeq \beta \circ p$ and there results a map $\varepsilon$ satisfying $\varepsilon \simeq \varepsilon^{\prime \prime} \circ \varepsilon^{\prime}$ and $r \circ \varepsilon=\beta \circ p$. Hence, $\varepsilon$ is a homotopy equivalence; since so is $\beta$, the induced map $\psi$ is also a homotopy equivalence. The last statement follows immediately from [16, Theorem 3].

2. Principal fibrations. A fibration $\mathscr{F}: F \rightarrow E \rightarrow B$ is principal if there are maps $\phi$ and $\varphi$ such that the diagram

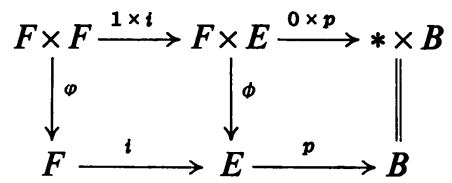

strictly commutes and $\varphi$ is an $H$-structure on $F$, i.e., $\varphi \mid F \vee F \simeq \nabla: F \vee F \rightarrow F$ where $\nabla$ is the folding map. This is the definition given in [18] with the third condition omitted. The maps $\varphi\left(\omega_{1}, \omega_{2}\right)=\omega_{1}+\omega_{2}$ and $\phi(\omega,(b, \eta))=(b, \omega+\eta)$, where + stands for path multiplication, obviously convert the strictly induced fibration (1) into a principal one; moreover, the action of $\Omega Y$ on $W$ is homotopy associative, i.e., $\phi \circ(\varphi \times 1) \simeq \phi \circ(1 \times \phi)$.

LEMMA 2.1. If $\mathscr{F}$ is a principal fibration, then homotopy-commutativity holds in the diagram

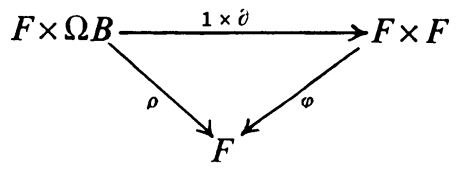

where $\rho$ is the operation, $\varphi$ the $H$-structure, and $\partial=\rho \mid * \times \Omega B$.

Proof. The top row in (4) is the Cartesian product of the two fibrations $F \rightarrow F \rightarrow *$ and $\mathscr{F}$. Therefore, it is a fibration and, by $[5,3.8]$, commutativity in (4) implies homotopy-commutativity in the square of the diagram

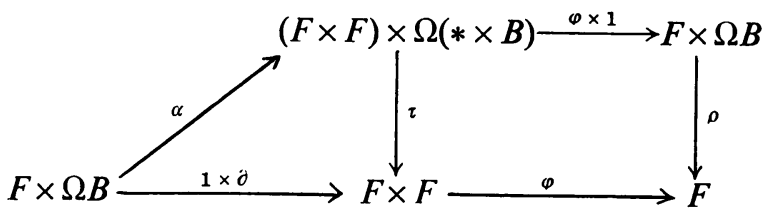

where $\tau$ is the operation in the product fibration. Inspection of the operation in a Cartesian product of fibrations reveals that $\tau$ is homotopic to the map $(x, y, *, \omega)$ $\rightarrow(x, \rho(y, \omega))$. The result follows upon defining $\alpha(x, \omega)=(x, *, *, \omega)$ so that $\tau \circ \alpha \simeq 1 \times \partial$ and $(\varphi \times 1) \circ \alpha \simeq 1$.

A map $\theta: F \rightarrow \Omega Y$ is primitive with respect to $\varphi$ if homotopy-commutativity holds in the diagram

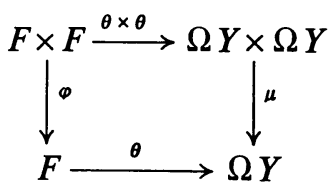


where $\mu$ is the loop multiplication. Unless the contrary is explicitly stated, we shall consider primitivity with respect to the $H$-structure $\varphi$ in a principal fibration.

TheOREM 2.2. Let $\mathscr{F}: F \rightarrow E \rightarrow B$ be a fibration in which $E$ and $B$ have the homotopy type of $C W$-complexes. Suppose that $B$ is $(m-1)$-connected and that $\pi_{q}(F) \neq 0$ only if $n \leqq q \leqq n+2 m-2$, where $m \geqq 1$ and $n \geqq 1$. If $\mathscr{F}$ is principal, and if there is a space $Y$ and a primitive homotopy equivalence $\theta: F \rightarrow \Omega Y$, then $\mathscr{F}$ is induced by some map $B \rightarrow Y$.

Proof. Consider the diagram

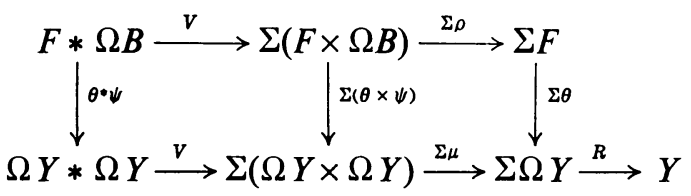

where $\psi=\theta \circ \partial$. The first square obviously commutes. Since $\mathscr{F}$ is principal and $\theta$ primitive, the second square homotopy-commutes by 2.1 and (5). According to [7, 2.4], the bottom composite is nullhomotopic. Therefore, the composite (2) is nullhomotopic and the result follows from 1.4.

We now turn to the study of conditions which guarantee that a fibration is principal. Recall that for any two spaces $A$ and $Z$, the set $\pi_{1}(A, Z)$ of based homotopy classes of maps $A \rightarrow \Omega Z$ is a group under the multiplication induced by loop multiplication. We shall also need the sequence

$$
\Omega X * \Omega Y \stackrel{w}{\longrightarrow} X b Y \stackrel{L}{\longrightarrow} X \vee Y \stackrel{J}{\longrightarrow} X \times Y \stackrel{\bullet}{\longrightarrow} X \# Y
$$

in which $J$ is the inclusion, $X \# Y$ results from $X \times Y$ by shrinking to a point the subset $X \vee Y$, and $Q$ is the identification map. The space $X b Y$ is the fiber of $J$, constructed as in (1), and it can readily be identified to $P X \times \Omega Y \cup \Omega X \times P Y$; the projection $L$ is then given by $L(\xi, \eta)=(\xi(1), \eta(1))$. The map $W$ is given by

$$
W((1-s) \xi \oplus s \eta)=\left(\xi_{\text {Min }(1,2-2 s)}, \eta_{\text {Min }(2 s, 1)}\right)
$$

where $\zeta_{u}(t)=\zeta(u t)$ for any path $\zeta$, and arguments based on the results in [16] reveal that $W$ is a homotopy equivalence if $X$ and $Y$ have the homotopy type of CW-complexes [7, p. 302].

The next result is, essentially, equivalent to [10,3.1 and 3.2]; the proof we give is slightly shorter and does not require $X$ and $Y$ to be 1-connected as in [10].

Proposition 2.3. For any map $f: X \rightarrow Y$ of spaces having the homotopy type of $C W$-complexes, the following two conditions are equivalent:

(i) The homomorphism $f_{1}: \pi_{1}(A, X) \rightarrow \pi_{1}(A, Y)$ induced by $f$ maps the first group into the center of the second for any space $A$;

(ii) The composite

$$
X \downarrow Y \stackrel{L}{\longrightarrow} X \vee Y \stackrel{f \vee 1}{\longrightarrow} Y \vee Y \stackrel{\nabla}{\longrightarrow} Y
$$

is nullhomotopic. 
Proof. Let $\gamma: \Omega X \times \Omega Y \rightarrow \Omega Y$ be the commutator map given by $\gamma(\xi, \eta)$ $=(f \circ \xi+\eta)+(-f \circ \xi-\eta)$, where + and - stand for loop multiplication and inversion. There is a map $\gamma^{\prime}: \Sigma(\Omega X \# \Omega Y) \rightarrow Y$ such that $\gamma^{\prime} \circ \Sigma Q$ is homotopic to the adjoint $\Sigma(\Omega X \times \Omega Y) \rightarrow Y$ of $\gamma$ [7, p. 313]. As shown in [7, 5.1], there is a homotopy $h_{t}: \Omega X * \Omega Y \rightarrow Y$ satisfying

$$
h_{0} \simeq \nabla \circ(f \vee 1) \circ L \circ W \quad \text { and } \quad h_{1} \simeq \gamma^{\prime} \circ \Sigma Q \circ V \text {, }
$$

where $V$ is the map described after 1.3. Since $\Sigma Q \circ V$ is a homotopy equivalence, one has $h_{1} \simeq 0$ if and only if $\gamma \simeq 0$. Since $W$ is a homotopy equivalence, the result follows upon noting that (i) holds if and only if $\gamma \simeq 0$.

If the conditions in 2.3 are fulfilled, we say that $f$ maps $\Omega X$ into the center of $\Omega Y$; this certainly happens if

(j) $\Omega Y$ is homotopy-commutative (e.g., if $Y$ is an H-space), or if

(jj) $f$ is nullhomotopic.

THEOREM 2.4. Let $\mathscr{F}: F \stackrel{i}{\longrightarrow} E \stackrel{p}{\longrightarrow} B$ be a fibration in which $E$ and $B$ have the homotopy type of $C W$-complexes. Suppose that $B$ is $(m-1)$-connected and that $\pi_{q}(F) \neq 0$ only if $n \leqq q \leqq n+2 l-2$, where $l=\operatorname{Min}(m, n)$ and $m \geqq 2, n \geqq 2$. If $i$ maps $\Omega F$ into the center of $\Omega E$, then $\mathscr{F}$ is principal and induced in each of the following cases:

(i) $\pi_{q}(B)=0$ for $q \geqq n+l$,

(ii) $\pi_{q}(B)=0$ for $q \geqq n+2 l-1$ and there is a map $\Gamma: \Omega B \rightarrow \Omega E$ such that $\Omega p \circ \Gamma=1$.

Proof. Consider the diagram

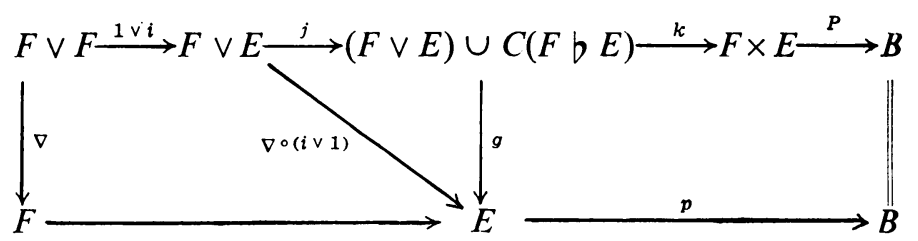

where the cone is attached by means of $L, j$ is the inclusion, $k$ is the obvious extension of $J: F \vee E \rightarrow F \times E$, and $P(x, a)=p(a)$ for $x \in F, a \in E$. Since $\Omega F$ maps into the center of $\Omega E$, the composite $\nabla \circ(i \vee 1) \circ L$ is nullhomotopic and there results a map $g$ yielding strict commutativity in the triangle. Therefore, $p \circ g \circ j=P \circ k \circ j$ and, by $[19,4.5]$, there is a map

$$
\beta: \Sigma(F b E) \rightarrow B \quad \text { with } \quad P \circ k \simeq(p \circ g) \uparrow \beta \quad \text { rel. } F \vee E,
$$

where $\mathrm{T}$ denotes the operation on the right of $\pi(\Sigma(F b E), D)$ on $\pi((F \vee E)$ $\cup C(F b E), D)$ for any space $D$. Since $\Sigma(F b E)$ is $(n+l-1)$-connected, $\beta$ is nullhomotopic if (i) holds, hence

$$
\beta \simeq p \circ \varepsilon
$$


where $\varepsilon: \Sigma(F b E) \rightarrow E$ is the constant map; if (ii) holds, (8) is certainly valid for some $\varepsilon$. Let $g_{0}=g \top \varepsilon$; then,

$$
g_{0} \circ j=g \circ j \quad \text { and } \quad P \circ k \simeq p \circ g_{0} \quad \text { rel. } F \vee E \text {, }
$$

where the second relation follows from (7) and (8). Since $p$ is a fiber map, $g_{0}$ extends to a homotopy

$$
g_{t}:(F \vee E) \cup C(F b E) \rightarrow E \text { with } p \circ g_{1}=P \circ k \text { and } p \circ g_{t} \circ d=* \text {, }
$$

where $d=j \circ(1 \vee i)$, and there results a homotopy $\nabla_{t}: F \vee F \rightarrow F$ satisfying $i \circ \nabla_{t}=g_{t} \circ d$ so that, by (9), $\nabla_{0}=\nabla$. Hence, we obtain the strictly commutative diagram

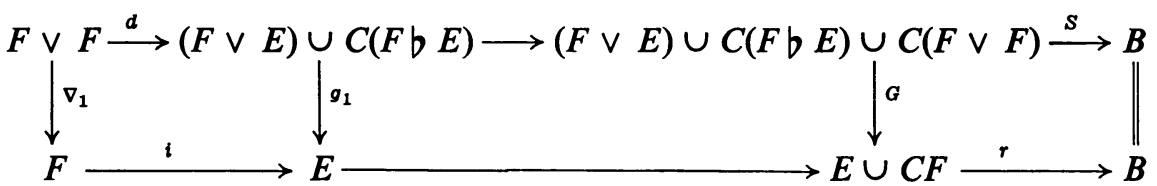

where $G$ is induced by $g_{1}$ and $\nabla_{1}, S$ and $r$ extend $P \circ k$ and $p$ by mapping the cones to the base-point, and $\nabla_{1} \simeq \nabla$. Next, since $k$ is $(n+2 l-1)$-connected and, clearly, $\pi_{q}(E)=0$ if $q \geqq n+2 l-1$, an obvious obstruction argument yields a map $\eta$ in the diagram

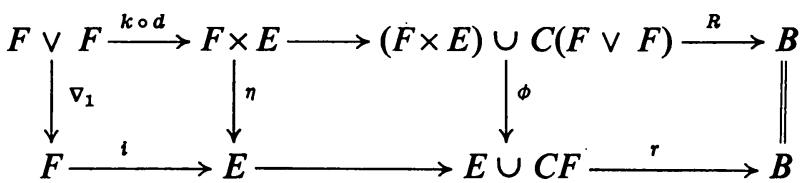

and a homotopy $H_{t}:(F \vee E) \cup C(F b E) \rightarrow E$ satisfying $H_{0}=g_{1}$ and $H_{1}=\eta \circ k$. Let $h_{t}=H_{t} \circ d$ so that $h_{0}=i \circ \nabla_{1}$ and $h_{1}=\eta \circ k \circ d$, let $\phi$ be induced by $\eta, \nabla_{1}, h_{t}$, and let $R$ extend $P$ by mapping the cone to the base-point. Let

$$
\Psi:(F \vee E) \cup C(F b E) \cup C(F \vee F) \rightarrow(F \times E) \cup C(F \vee F)
$$

be the map induced by $k$. Obviously, $S=R \circ \Psi$; also, it is easily seen that $\phi \circ \Psi \simeq G$. Therefore, $r \circ \phi \circ \Psi \simeq R \circ \Psi$. Since $k$ is $(n+2 l-1)$-connected, so is $\Psi$ by the five lemma and, since $\pi_{q}(B)=0$ if $q \geqq n+2 l$, a classical obstruction argument reveals that $r \circ \phi \simeq R$. Therefore, 1.1 applied to the preceding diagram yields a strictly commutative diagram

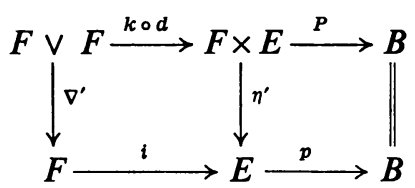

with $\nabla^{\prime} \simeq \nabla_{1}$, hence $\nabla^{\prime} \simeq \nabla$, and $\eta^{\prime} \simeq \eta$. Since $F \times F$ is the fiber of $P, \nabla^{\prime}$ extends to a map $\varphi: F \times F \rightarrow F$ such that $i \circ \varphi^{\prime}=\eta^{\prime} \circ(1 \times i)$, and $\mathscr{F}$ is proved to be principal. Since $l \leqq n$, it follows from [13] that there is a space $Y$ and a primitive homotopy 
equivalence $F \rightarrow \Omega Y$ and, since $l \leqq m$, it follows from 2.2 that $\mathscr{F}$ is an induced fibration.

COROLlaRY 2.5. Suppose that $\pi_{q}(B) \neq 0$ only if $m \leqq q \leqq n+m-1$ and that $\pi_{q}(F) \neq 0$ only if $n \leqq q \leqq n+m-1$, where $n \geqq m \geqq 2$. If the Whitehead product pairing $W: \pi_{n}(F) \otimes \pi_{m}(E) \rightarrow \pi_{n+m-1}(E)$ induced by $i$ vanishes, then $\mathscr{F}$ is principal and induced.

Proof. The homomorphism $D_{n+m-1}$ of $(n+m-1)$-dimensional homotopy groups induced by the composite

$$
D: F b E \stackrel{L}{\longrightarrow} F \vee E \stackrel{i \vee 1}{\longrightarrow} E \vee E \stackrel{\nabla}{\longrightarrow} E
$$

is well known to vanish if and only if $W=0$. Since $F b E$ is $(n+m-2)$-connected and $\pi_{q}(E)=0$ for $q=1$ and for $q \geqq n+m$, an easy obstruction argument reveals that $D$ is nullhomotopic if and only if $D_{n+m-1}=0$, and the result follows from 2.4(i).

REMARK 2.6. It is easily seen that the conditions imposed on $i$ in 2.4 and, hence, on $W$ in 2.5 are necessary for $\mathscr{F}$ to be induced without any connectivity assumptions.

Under appropriate conditions, 2.5 extends by one unit the range of applicability of 1.5. The case $n>m$ of 2.5 applies to the study of Postnikov decompositions of spaces, and strengthens the result in $[15,5.6]$.

Example 2.7. Consider the fibration $S^{1} \rightarrow S^{2 r+1} \rightarrow C P^{r}$, where $C P^{r}$ is the complex projective $r$-space. It is well known, and it also follows from 1.5, that the fibration is induced. Hence we obtain a triple $S^{2 r+1} \rightarrow C P^{r} \rightarrow K(Z, 2)$ which we may regard as a fibration; as usual, $K(\pi, n)$ stands for an Eilenberg-MacLane space. Let $X(n)$ result by killing off the homotopy groups of $X$ in dimensions $>n$. We examine the fibrations

$$
\mathscr{F}_{q}: S^{2 r+1}(2 r+1+q) \rightarrow C P^{r}(2 r+1+q) \rightarrow K(Z, 2) \text { for } 0 \leqq q \leqq 2 .
$$

According to $1.5, \mathscr{F}_{0}$ is certainly induced. Let

$$
W: \pi_{2 r+1}\left(S^{2 r+1}\right) \otimes \pi_{2}\left(C P^{r}\right) \rightarrow \pi_{2 r+2}\left(C P^{r}\right)
$$

be the Whitehead product pairing. If $r$ is even, $W$ is nonvanishing [1] so that, by 2.6, $\mathscr{F}_{q}$ is not induced when $q \geqq 1$. If $r$ is odd, $W=0$ [1] so that, by $2.5, \mathscr{F}_{1}$ is induced; moreover, it is easily seen that $\Omega S^{2 r+1}(2 r+3)$ maps into the center of $\Omega C P^{r}(2 r+3)$ so that, by 2.4 , also $\mathscr{F}_{2}$ is induced.

The next two examples show that the connectivity assumptions in 2.4(i) are the best possible.

EXAMPLE 2.8. Let $F=C P^{2}(5)$; then, $\Omega F$ is homotopy-commutative but $F$ fails to be an $H$-space [2, 3.10]. Hence, the fibration $F \rightarrow F \rightarrow *$ is not induced; all conditions in 2.4(i) are fulfilled except that $\pi_{q}(F) \neq 0$ only if $n \leqq q \leqq n+2 l-1$.

EXAMPLE 2.9. Let $Q$ be the additive group of rationals and let $n \geqq 4$ be even. Then $K(Q, n-1)$ and the Moore space $K^{\prime}(Q, n-1)$ have the same homotopy 
type. Therefore, by $(6), K(Q, n) b K(Q, n)$ has the homotopy type of $K(Q, 2 n-1)$ and, by 1.5 , the fibration

$$
K(Q, 2 n-1) \rightarrow K(Q, n) \vee K(Q, n) \rightarrow K(Q, n) \times K(Q, n)
$$

is induced. The resulting fibration

$$
K(Q, n) \vee K(Q, n) \rightarrow K(Q, n) \times K(Q, n) \rightarrow K(Q, 2 n)
$$

is not induced since $K(Q, n) \vee K(Q, n)$ is not an $H$-space [9]; all conditions in 2.4(i) are fulfilled except that $\pi_{q}(B) \neq 0$ only if $m \leqq q \leqq n+l$.

We close this section by giving a simple proof of a known result on fibrations of aspherical spaces, i.e., spaces with vanishing homotopy groups in dimensions $\geqq 2$; a semisimplicial proof can be found in $[11,5.4]$.

THEOREM 2.10. Let $\mathscr{F}: F \stackrel{i}{\longrightarrow} E \stackrel{p}{\longrightarrow} B$ be a fibration in which all spaces have the homotopy type of aspherical $C W$-complexes. If the homomorphism $i_{1}: \pi_{1}(F) \rightarrow \pi_{1}(E)$ maps $\pi_{1}(F)$ into the center of $\pi_{1}(E)$, then $\mathscr{F}$ is induced by some map of $B$ into an Eilenberg-MacLane space $K\left(\pi_{1}(F), 2\right)$.

Proof. Since $\pi_{1}(F)$ lies in the center of $\pi_{1}(E)$, the function $\phi_{1}: \pi_{1}(F) \times \pi_{1}(E)$ $\rightarrow \pi_{1}(E)$ given by $\phi_{1}(u, v)=i_{1}(u)+v$ is a homomorphism. Therefore, since $E$ is aspherical, there is a map $\phi: F \times E \rightarrow E$ which induces $\phi_{1}$. Obviously, one has $p_{1} \circ \phi_{1}=(0 \times p)_{1}$ so that, since $B$ is aspherical, the right-hand square in (4) homotopy-commutes. Since $p$ is a fiber map, we may assume that it strictly commutes. There results a map $\varphi$ which is an $H$-structure on $F$ since the homomorphism $\varphi_{1}: \pi_{1}(F) \times \pi_{1}(F) \rightarrow \pi_{1}(F)$ is the identity on each factor. Since $F$ may carry at most one $H$-structure, any homotopy equivalence $F \rightarrow \Omega K\left(\pi_{1}(F), 2\right)$ is primitive, and the result follows from 2.2 .

3. Induced cofibrations. A triple $\mathscr{C}: A \stackrel{d}{\longrightarrow} X \stackrel{f}{\longrightarrow} C$ is a cofibration if $d$ is an inclusion map with the homotopy extension property and $C$ results from $X$ by shrinking the subset $A$ to a point; $f$ is the identification map. For any map $f: X \rightarrow Y$ we denote by $E_{f}$ and $F_{f}$ the mapping track and the fiber of $f$ given, as in (1), by

$$
E_{f}=\left\{(x, \eta) \in X \times Y^{I} \mid f(x)=\eta(1)\right\}, \quad F_{f}=\{(x, \eta) \in X \times P Y \mid f(x)=\eta(1)\} ;
$$

the map $h: X \rightarrow E_{f}$ given by $h(x)=\left(x, \eta_{x}\right)$ with $\eta_{x}(s)=f(x)$ is a homotopy equivalence, and the map $p: E_{f} \rightarrow Y$ given by $p(x, \eta)=\eta(0)$ is a fiber map satisfying $p \circ h=f$. If $f$ is a fiber map, the map $f^{-1}(*) \rightarrow F_{f}$ defined by $h$ is a homotopy equivalence, so that the introduction of $F_{f}$ causes no real ambiguity. In any triple $\mathscr{C}$ satisfying $f \circ d=*$, the map $d$ lifts to the map $e: A \rightarrow F_{f}$ given by $e(a)=(d(a), *)$. The dual of 1.1 may now easily be formulated and proved noting that the mapping track is dual to the mapping cylinder.

For any cofibration $\mathscr{C}$ we may construct a sequence of cofibrations

$$
\mathscr{C}_{k}: A \stackrel{d_{k}}{\longrightarrow} X_{k} \stackrel{f_{k}}{\longrightarrow} C_{k} \quad(k \geqq 0)
$$


as follows: $\mathscr{C}_{0}=\mathscr{C}, e_{k+1}: A \rightarrow F_{k+1}$ lifts $d_{k}$ to the fiber $F_{k+1}$ of $f_{k}$, and $d_{k+1}$ results by converting $e_{k+1}$ into a homotopically equivalent cofiber map. If $X$ and $A$ have the homotopy type of $\mathrm{CW}$-complexes, we may use the Blakers-Massey theorem to compute the connectivity of every $e_{k}$.

Proposition 3.1. Suppose that $A$ is $(m-1)$-connected, $C$ is $n$-connected, and that $Y$ is a $C W$-complex of dimension $\leqq n+(k+1)(m-1)$, where $m \geqq 2, n \geqq 1$. For any map $f: Y \rightarrow X_{k}$ with $f_{k} \circ f \cong 0$ there is a map $g: Y \rightarrow A$ with $d_{k} \circ g \simeq f$.

Proof. Since $f_{k} \circ f \simeq 0, f$ lifts to a map $h: Y \rightarrow F_{k+1}$. Since $e_{k+1}$ is $(n+(k+1)(m-1))$ connected, the restriction on the dimension of $Y$ yields a map $g: Y \rightarrow A$ satisfying $e_{k+1} \circ g \simeq h$.

The result in 1.2 suggests, by duality, that the homotopy type of $C_{k+1}$ is determined by those of $C_{k}$ and $\Sigma A$. However, an example due to M. G. Barratt [7, 3.5] disproves this conjecture. Nevertheless, according to [7, 4.1], there is an $(N-1)$ connected map $\mathscr{T}: C_{1} \rightarrow \Omega(C b \Sigma A)$, where $N=m+n+\operatorname{Min}(m, n)$.

COROLLARY 3.2. If $H_{N-2}(Y)$ is free and $H_{q}(Y)=0$ for $q \geqq N-1$, then for any map $f: Y \rightarrow X_{1}$ with $\mathscr{T} \circ f_{1} \circ f \simeq 0$ there is a map $g: Y \rightarrow A$ with $d_{1} \circ g \simeq f$.

Proof. Let $Z$ and $\varphi: Y \rightarrow Z$ result by shrinking to a point the 1 -skeleton of $Y$. Then, $Z$ is 1-connected and, since $N \geqq 4, H_{N-2}(Z)$ is free and $H_{q}(Z)=0$ for $q \geqq N-1$ so that $Z$ has the homotopy type of a CW-complex of dimension $\leqq N-2$. Since $X_{1}$ is 1-connected, there is a map $\psi: Z \rightarrow X_{1}$ with $\psi \circ \varphi \simeq f$ and, since $\Omega(C b \Sigma A)$ is at least 2-connected, $\mathscr{T} \circ f_{1} \circ \psi \simeq 0$. Since $\operatorname{dim} Z \leqq N-2, f_{1} \circ \psi \simeq 0$ and, by 3.1 with $k=1$, there is a map $\gamma: Z \rightarrow A$ with $d_{1} \circ \gamma \simeq \psi$. The map $g$ results by setting $g=\gamma \circ \varphi$.

In any cofibration $\mathscr{C}$, the suspension $\Sigma A$ cooperates [5] on the cofiber $C$ through a map $\tau: C \rightarrow C \vee \Sigma A$. The Hopf invariant of $\mathscr{C}[7, \S 4]$ is the composite

$$
\mathscr{H}: \Omega C \stackrel{\Omega \tau}{\longrightarrow} \Omega(C \vee \Sigma A) \stackrel{T}{\longrightarrow} \Omega(C b \Sigma A)
$$

where, with the notation introduced in (6), the homotopy class of $T: \Omega(X \vee Y)$ $\rightarrow \Omega(X b Y)$ is uniquely determined by the equation $\Omega L \circ T+M \circ \Omega J \simeq 1$ in which

$$
\begin{aligned}
& M: \Omega(X \times Y) \rightarrow \Omega(X \vee Y) \text { is given by } M(\xi, \eta)(s)=(*, \eta(2 s)) \\
& =(\xi(2 s-1), *) \text {. }
\end{aligned}
$$

We describe two cofibrations $\mathscr{C}$ and $\mathscr{C}^{\prime}$ over the same cobase $A$ as equivalent if there is a homotopy equivalence $\xi: X \rightarrow X^{\prime}$ satisfying $\xi \circ d=d^{\prime}$; there exists then a homotopy inverse $\xi^{\prime}$ of $\xi$ satisfying $\xi^{\prime} \circ d^{\prime}=d$. A cofibration $\mathscr{C}$ is induced if there is a space $Y$ and a map $g: Y \rightarrow A$ such that $\mathscr{C}$ is equivalent to the cofibration strictly induced by $g$ from $Y \rightarrow C Y \rightarrow \Sigma Y$, i.e., to the triple

$$
A \rightarrow A \cup_{g} C Y \rightarrow \Sigma Y
$$


in which the first map is the obvious inclusion and the second shrinks $A$ to a point; it then follows that $C$ has the homotopy type of $\Sigma Y$.

For any 1-connected CW-complex $K$ we write $\operatorname{dim} K \leqq n$ to indicate that $H_{n}(K)$ is free and $H_{q}(K)=0$ if $q>n$. The map $S$ below is given by $S(y)(s)=\langle 1-s, y\rangle$.

THEOREM 3.3. Let

$$
\mathscr{C}: A \stackrel{d}{\longrightarrow} X \stackrel{f}{\longrightarrow} C
$$

be a cofibration in which $(X, A)$ is a CW-pair. Suppose that $A$ is $(m-1)$-connected, $C$ is n-connected, and $\operatorname{dim} C \leqq n+m+\operatorname{Min}(m, n)-1$, where $m \geqq 2, n \geqq 1$. If there is a $C W$-complex $Y$ and a homotopy equivalence $\theta: \Sigma Y \rightarrow C$ such that the composite

$$
Y \stackrel{s}{\longrightarrow} \Omega \Sigma Y \stackrel{\Omega \theta}{\longrightarrow} \Omega C \stackrel{\mathscr{H}}{\longrightarrow} \Omega(C b \Sigma A)
$$

is nullhomotopic, then $\mathscr{C}$ is induced by some map $g: Y \rightarrow A$.

Proof. Dualize the proof of 1.4 noting that, according to $[7,4.1], \mathscr{H}$ is homotopic to the composite

$$
\Omega(C b \Sigma A) \stackrel{\mathscr{T}}{\longleftarrow} C_{1} \stackrel{f_{1}}{\longleftarrow} X_{1} \stackrel{u}{\longleftarrow} F_{1} \stackrel{\partial}{\longleftarrow} \Omega C
$$

in which $\partial(\omega)=(*, \omega)$ and $u$ is the homotopy equivalence obtained when converting $e_{1}$ into $d_{1}$ so that $d_{1} \simeq u \circ e_{1}$.

We derive the known [8], [12]

Corollary 3.4. If $A$ is $(m-1)$-connected, $C$ is $n$-connected, $\operatorname{dim} C \leqq n+m$ $(m \geqq 2, n \geqq 1)$, and if there is a homotopy equivalence $\theta: \Sigma Y \rightarrow C$, then $\mathscr{C}$ is induced by some map $g: Y \rightarrow A$.

Proof. $\Omega(C b \Sigma A)$ is $(n+m-1)$-connected and $H^{q}(Y ; G)=0$ for all $G$ if $q \geqq n+m$ so that (11) is certainly nullhomotopic.

Remark 3.5. The homotopy equivalence $\Sigma Y \rightarrow C$ resulting in 3.1 and 3.4 from the fact that $\mathscr{C}$ is induced by $g: Y \rightarrow A$ is homotopic to the original homotopy equivalence $\theta: \Sigma Y \rightarrow C$.

The next result was first obtained in [4] by means of an elaborate argument which requires $X$ to have finitely generated homology. The proof we give dispenses with this assumption and relies only on 3.4 and on a result in [4] concerning homology decomposition. Recall that $X$ is an $H^{\prime}$-space if there is a map $\varphi: X \rightarrow X \vee X$ such that the composite $J \circ \varphi: X \rightarrow X \times X$ is homotopic to the diagonal map $\Delta$. A map $\theta: \Sigma Y \rightarrow X$ is primitive with respect to $\varphi$ if homotopy-commutativity holds in the diagram

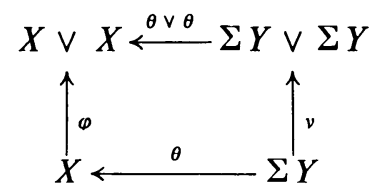

$$
\text { where } \begin{aligned}
\nu\langle s, y\rangle & =(\langle 2 s, y\rangle, *) \\
& =(*,\langle 2 s-1, y\rangle)
\end{aligned}
$$

defines the comultiplication on the suspension. 
Proposition 3.6. Let $X$ be an $(n-1)$-connected $C W$-complex such that $H_{3 n-3}(X)$ is free and $H_{q}(X)=0$ for $q>3 n-3(n \geqq 1)$. If $X$ is an $H^{\prime}$-space, then there is a $C W$ complex $Y$ and a primitive homotopy equivalence $\theta: \Sigma Y \rightarrow X$.

Proof. Let $E_{J}$ be the mapping track of $J: X \vee X \rightarrow X \times X$. As is well known, inspection of the fibration induced by $\Delta: X \rightarrow X \times X$ from $E_{J} \rightarrow X \times X$ reveals that there is a map $\Gamma$ yielding homotopy-commutativity in the diagrams
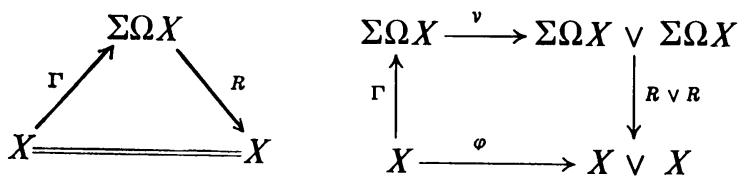

where $R\langle s, \omega\rangle=\omega(s)$ and $\nu$ is the comultiplication. Now, if $n=1$, then $X$ is contractible; for, the fundamental group of an $H^{\prime}$-CW-complex is always free (non-Abelian) and $H_{1}(X)=0$ then implies $\pi_{1}(X)=0$. Let $n \geqq 2$. Let $W$ be the $(3 n-4)$-skeleton of $\Omega X$ with inclusion $f: W \rightarrow \Omega X ; \Omega X$ may be assumed to be a CW-complex according to [16]. Since $\Sigma f$ is $(3 n-3)$-connected and $H^{q}(X ; G)=0$ for all $G$ if $q>3 n-3$, there is a map $\gamma: X \rightarrow \Sigma W$ such that $\Sigma f \circ \gamma \simeq \Gamma$. Consider the cofibration

$$
X \stackrel{\gamma}{\longrightarrow} \Sigma W \longrightarrow \Sigma W \cup_{\gamma} C X
$$

and note that $R \circ \Sigma f \circ \gamma \simeq 1$ so that $\gamma_{*}$ is monomorphic in both homology and homotopy. Since $R$ is $(2 n-1)$-connected, $\Gamma$ and hence $\gamma$ are $(2 n-2)$-connected, and it follows that $\Sigma W \cup C X$ is $(2 n-2)$-connected; also, the homology sequence of (14) reveals that $H_{q}(\Sigma W \cup C X)=0$ if $q>3 n-3$. Since any $(p-1)$-connected CW-complex $K$ with $H_{2 p-1}(K)$ free and $H_{q}(K)=0$ for $q \geqq 2 p$ has the homotopy type of a suspension if $p \geqq 2, \Sigma W \cup C X$ has the homotopy type of some suspension $\Sigma A$ and 3.4 now implies that (14) is induced by some map $\xi: A \rightarrow X$. Hence, we obtain a cofibration in the top row of the diagram

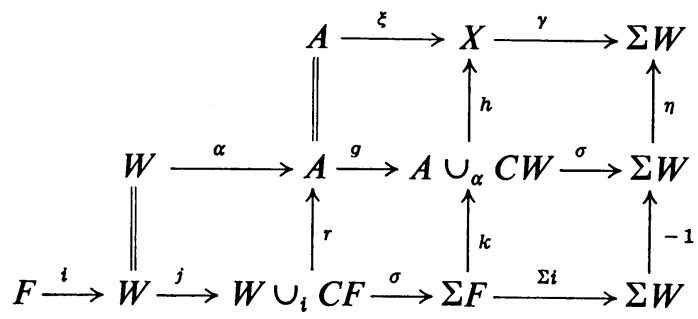

As in [3, p. 443] we may assume $A$ to be 1-connected, and 3.4 then readily implies that this cofibration is, in turn, induced by some map $\alpha$. Hence, there are homotopy equivalences $h$ and $\eta$ yielding commutativity in the upper squares, where $g$ is the inclusion and $\sigma$ shrinks $A$ to a point. Moreover, by 3.5 ,

$$
\eta \simeq 1
$$


Next, let $F$ be the fiber of $\alpha$ with projection $i$, let $j$ be the inclusion, let $r$ be the obvious extension of $\alpha$, and let $\sigma$ shrink $W$ to a point. There results a map $k$ yielding homotopy-commutativity in its two adjacent squares, where $-1\langle s, w\rangle=\langle 1-s, w\rangle$ (see, e.g., [7, 1.6]). By the Serre theorem, $r$ is homology $(3 n-3)$-connected and the five lemma implies the same for $k$. Next, the result on homology decomposition given in $[4,2.1]$ yields a connected $\mathrm{CW}$-complex $Y$ and a map $\phi: Y \rightarrow F$ such that $\theta^{\prime}=h \circ k \circ \Sigma \phi: \Sigma Y \rightarrow X$ induces isomorphisms of homology groups in all dimensions. Since $\Sigma Y$ and $X$ are 1-connected, $\theta^{\prime}$ is a homotopy equivalence and so is also $\theta=\theta^{\prime} \circ(-1)$. Finally, in order to prove that $\theta$ is primitive it clearly suffices to prove it for $h \circ k \circ(-1)$. But homotopy-commutativity in (15) yields

$$
\gamma \circ h \circ k \circ(-1) \simeq \eta \circ \Sigma i
$$

and the result follows from (16), (13), and the primitivity of $\Sigma f$ and $\Sigma i$ with respect to comultiplication.

REMARK 3.7. The second result in [4] is also valid without assuming any homology to be finitely generated; for, the map $\psi$ used in the proof of Theorem B in [4] has the right connectivity according to [7, 4.1]. Also, the method of proof used in 3.6 above yields an alternative proof of Lemma 3.6 in [3].

4. Principal cofibrations. A cofibration $\mathscr{C}: A \rightarrow X \rightarrow C$ is principal if there are maps $\phi$ and $\varphi$ such that the diagram

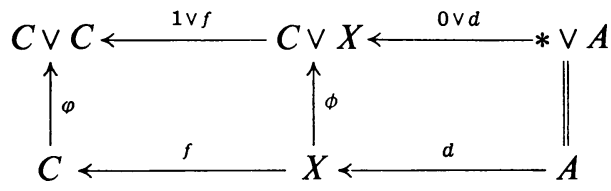

strictly commutes and $\varphi$ is an $H^{\prime}$-structure on $C$. A strictly induced cofibration is principal. From now on we shall only consider primitivity with respect to the $H^{\prime}$ structure $\varphi$ in a principal cofibration.

LEMMA 4.1. If $\mathscr{C}$ is a principal cofibration, then homotopy-commutativity holds in the diagram

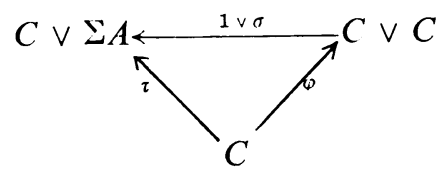

where $\tau$ is the cooperation, $\varphi$ the $H^{\prime}$-structure, and $\sigma$ the composite

$$
C \stackrel{\imath}{\longrightarrow} C \vee \Sigma A \stackrel{\mathrm{pr}}{\longrightarrow} \Sigma A
$$

in which $\mathrm{pr}$ is the projection.

Theorem 4.2. Let $\mathscr{C}: A \rightarrow X \rightarrow C$ be a cofibration in which $(X, A)$ is a $C W$-pair. Suppose that $A$ is $(m-1)$-connected, $C$ is $n$-connected, and $\operatorname{dim} C \leqq n+m+\operatorname{Min}(m, n)$ -1 , where $m \geqq 2, n \geqq 1$. If $\mathscr{C}$ is principal and if there is a $C W$-complex $Y$ and $a$ primitive homotopy equivalence $\theta: \Sigma Y \rightarrow C$, then $\mathscr{C}$ is induced by some map $Y \rightarrow A$. 
Proof. With $\psi=\sigma \circ \theta$, the diagram

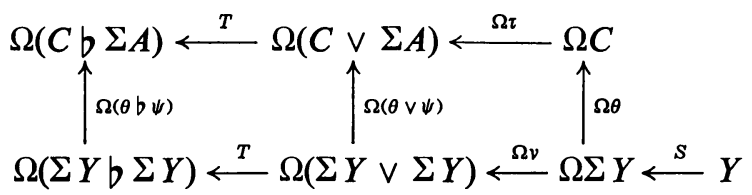

homotopy-commutes. The bottom row is nullhomotopic by [7, 4.1]. Therefore, (11) is nullhomotopic and 3.3 implies the result.

We now turn to the study of conditions which guarantee that a cofibration is principal. Recall that for any two spaces $Z$ and $B$, the set $\pi_{1}(Z, B)$ of based homotopy classes of maps $\Sigma Z \rightarrow B$ is a group under the multiplication induced by suspension comultiplication.

Proposition 4.3. For any map $f: X \rightarrow Y$ of spaces having the homotopy type of connected $C W$-complexes, the following two conditions are equivalent:

(i) The homomorphism $f^{1}: \pi_{1}(Y, B) \rightarrow \pi_{1}(X, B)$ induced by $f$ maps the first group into the center of the second for any space $B$;

(ii) The composite

$$
X \stackrel{\Delta}{\longrightarrow} X \times X \stackrel{f \times 1}{\longrightarrow} Y \times X \stackrel{Q}{\longrightarrow} Y \# X \stackrel{s}{\longrightarrow} \Omega \Sigma(Y \# X),
$$

where $S(z)(s)=\langle s, z\rangle$, is nullhomotopic.

The proof is similar to that given in [10, 4.1]. Note that conditions 2.3(ii) and 4.3(ii) are not precise duals, owing to the presence of the map $S$ in the latter.

If the conditions in 4.3 are fulfilled, we say that $f$ maps $\Sigma X$ into the cocenter of $\Sigma Y$; this certainly happens if

(j) $\Sigma X$ is homotopy-commutative (e.g., if $X$ is an $H^{\prime}$-space), or if

(jj) $f$ is nullhomotopic.

THEOREM 4.4. Let

$$
\mathscr{C}: A \stackrel{d}{\longrightarrow} X \stackrel{f}{\longrightarrow} C
$$

be a cofibration in which $(X, A)$ is a CW-pair. Suppose that $A$ is $(m-1)$-connected, $C$ is $n$-connected, and that $\operatorname{dim} C \leqq n+\operatorname{Min}(2 m-1,2 n)$, where $m \geqq 2, n \geqq 1$. If $f$ maps $\Sigma X$ into the cocenter of $\Sigma C$, then $\mathscr{C}$ is principal and induced in each of the following cases:

(i) $\operatorname{dim} A \leqq n+\operatorname{Min}(m-1, n)$,

(ii) $\operatorname{dim} A \leqq n+\operatorname{Min}(2 m-2,2 n)$ and there is a map $\Gamma: \Sigma X \rightarrow \Sigma A$ such that $\Gamma \circ \Sigma d=1$.

Proof. Dualize the proof of 2.4 noting that comparison of $\operatorname{dim} X$ with the connectivity of $S$ in 4.3(ii) implies that the composite

is nullhomotopic.

$$
X \stackrel{\Delta}{\longrightarrow} X \times X \stackrel{f \times 1}{\longrightarrow} C \times X \stackrel{Q}{\longrightarrow} C \# X
$$


We derive the following supplement of 3.4 , in which the pairing of the coefficient groups is given by the natural isomorphism

$$
\pi_{n+1}(C) \otimes \pi_{m}(X) \rightarrow \pi_{n+m+1}(C \# X)
$$

resulting from the Künneth formula and the Hurewicz isomorphism.

Corollary 4.5. Suppose that $A$ is (m-1)-connected, $C$ is n-connected, $\operatorname{dim} A \leqq n+m-1$, and $\operatorname{dim} C \leqq n+m+1$, where $n \geqq m-1$ and $m \geqq 2, n \geqq 2$. If the cup-product pairing

$$
H^{n+1}\left(C ; \pi_{n+1}(C)\right) \otimes H^{m}\left(X ; \pi_{m}(X)\right) \rightarrow H^{n+m+1}\left(X ; \pi_{n+m+1}(C \# X)\right)
$$

induced by $f$ vanishes, then $\mathscr{C}$ is principal and induced.

Proof. Since $n \geqq 2$, the dimension assumptions in 4.4(i) are fulfilled, and it remains to show that $f$ maps $\Sigma X$ into the cocenter of $\Sigma C$. Hence, by 4.3 , it suffices to prove that the composite

$$
\phi: X \stackrel{\Delta}{\longrightarrow} X \times X \stackrel{f \times 1}{\longrightarrow} C \times X \stackrel{Q}{\longrightarrow} C \# X
$$

is nullhomotopic. Since $C \# X$ is $(n+m)$-connected and $\operatorname{dim} X \leqq n+m+1$, one has $\phi \simeq 0$ if and only if $\phi^{n+m+1}$ maps the fundamental class of $C \# X$ into 0 . The fundamental class clearly lies in the image of

$$
H^{n+1}\left(C ; \pi_{n+1}(C)\right) \otimes H^{m}\left(X ; \pi_{m}(X)\right) \rightarrow H^{n+m+1}\left(C \# X ; \pi_{n+m+1}(C \# X)\right),
$$

and the result follows from the interpretation of the cup-product in terms of the diagonal map.

REMARK 4.6. It is easy to see that the condition imposed on $f$ in 4.4 is necessary for $\mathscr{C}$ to be induced under no further assumptions; since the map $S$ in 4.3(ii) induces epimorphisms of cohomology groups in all dimensions, it follows that also the vanishing of the cup-product pairing

$$
H^{*}\left(C ; G^{\prime}\right) \otimes H^{*}\left(X ; G^{\prime \prime}\right) \rightarrow H^{*}(X ; G) \quad \text { for any pairing } G^{\prime} \otimes G^{\prime \prime} \rightarrow G
$$

of Abelian groups is necessary for $\mathscr{C}$ to be induced.

A dual of $[15,5.6]$ is expressed by the next result which follows from 4.5 and 4.6 noting that $f$ induces an epimorphism of $(n+1)$-dimensional cohomology groups.

Proposition 4.7. Let $X$ be an $(m-1)$-connected $C W$-complex of dimension $\leqq n+m+1$, and let $A$ be its $n$-skeleton, where $n \geqq m \geqq 2$. Then, the cofibration

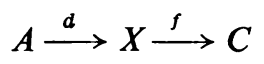

is induced if and only if the cup-product pairing $H^{n+1}\left(X ; G^{\prime}\right) \otimes H^{m}\left(X ; G^{\prime \prime}\right)$ $\rightarrow H^{n+m+1}(X ; G)$ vanishes for any pairing $G^{\prime} \otimes G^{\prime \prime} \rightarrow G$ of Abelian groups.

We conclude by invoking a classical example in order to show that the condition on $\operatorname{dim} A$ in 4.4(i), even though quite restrictive, is the best possible. 
EXAMPLe 4.8. Let $A=S^{7}, X=S^{4}$, and let $d$ be the Hopf map, so that $C$ is the quaternionic projective plane. Then, $f$ maps $\Sigma X$ into the cocenter of $\Sigma C$ since $\Sigma X$ is homotopy-commutative (see (j) after 4.3) and all conditions in 4.4(i) are fulfilled except that $\operatorname{dim} A=n+\operatorname{Min}(m-1, n)+1$. The cofibration is not induced since $C$ has a nonvanishing cup-product with coefficients $Z_{2}$ and, therefore, fails to have the homotopy type of a suspension.

\section{REFERENCES}

1. M. G. Barratt, I. M. James and N. Stein, Whitehead products and projective spaces, J. Math. Mech. 9 (1960), 813-819.

2. I. Berstein and T. Ganea, Homotopical nilpotency, Illinois J. Math. 5 (1961), 99-130.

3. I. Berstein and P. J. Hilton, Category and generalized Hopf invariants, Illinois J. Math. 4 (1960), 437-451.

4. - On suspensions and comultiplications, Topology 2 (1963), 73-82.

5. B. Eckmann and P. J. Hilton, Operators and cooperators in homotopy theory, Math. Ann. 141 (1960), 1-21.

6. T. Ganea, Fibrations and cocategory, Comment. Math. Helv. 35 (1961), 15-24.

7. - A generalization of the homology and homotopy suspension, Comment. Math. Helv. 39 (1965), 295-322.

8. — Upper estimates for the Lyusternik-Šnirel'man category, Dokl. Akad. Nauk SSSR 136 (1961), 1273-1276 = Soviet Math. Dokl. 2 (1961), 180-183.

9. T. Ganea and P. J. Hilton, On the decomposition of spaces in Cartesian products and unions, Proc. Cambridge Philos. Soc. 55 (1959), 248-256.

10. T. Ganea, P. J. Hilton and F. P. Peterson, On the homotopy-commutativity of loop spaces and suspensions, Topology 1 (1962), 133-141.

11. P. J. Hilton, On a generalization of nilpotency to semisimplicial complexes, Proc. London Math. Soc. 10 (1960), 604-622.

12. - On excision and principal fibrations, Comment. Math. Helv. 35 (1961), 77-84.

13. - Remark on loop spaces, Proc. Amer. Math. Soc. 15 (1964), 596-600.

14. W. Massey, Some problems in algebraic topology and the theory of fibre bundles, Ann. of Math. 62 (1955), 327-359.

15. J. P. Meyer, Principal fibrations, Trans. Amer. Math. Soc. 107 (1963), 177-185.

16. J. Milnor, On spaces having the homotopy type of $\mathrm{CW}$-complexes, Trans. Amer. Math. Soc. 90 (1959), 272-280.

17. Y. Nomura, A generalization of suspension theorems, Nagoya Math. J. 19 (1961), 159-167.

18. F. P. Peterson and E. Thomas, A note on nonstable cohomology operations, Bol. Soc. Mat. Mexicana 3 (1958), 13-18.

19. D. Puppe, Homotopiemengen und ihre induzierten Abbildungen. I, Math. Z. 69 (1958), 299-344.

UNIVERSITY OF WASHINGTON,

Seattle, Washington 